\title{
USING THE FUTURE L-BAND COMMUNICATION SYSTEM FOR NAVIGATION
}

\author{
Michael Schnell, Ulrich Epple and Felix Hoffmann \\ German Aerospace Center (DLR), Oberpfaffenhofen, Germany
}

\begin{abstract}
In this paper, we propose to apply the future Lband digital aeronautical communication system not only for communications but also for navigation purposes. In particular, the future communication system might be used as alternative positioning, navigation, and timing means in case other navigation means, e.g. satellite based navigation, are temporally not available due to intentional or unintentional interference. The approach how navigation is achieved using the future communication system is described and first results about the feasibility of this approach are presented. The accuracy of the aircraft positioning estimates turns out to be in the order of 1 to $100 \mathrm{~m}$, depending on the number and geographic distribution of the ground stations used for determining the underlying ranging estimates. Considering the horizontal aircraft position only, an even higher accuracy is achieved, since the ground stations are located at comparable altitude leading to a dominant position error in the vertical component. These promising first results lead to the conclusion that navigation with the future communication system is a very interesting approach for the alternative positioning, navigation, and timing system required as fallback solution for satellite-based navigation.
\end{abstract}

\section{Introduction}

Currently, aeronautical communications and navigation are undergoing a major renovation process to assist the modernization of Air-Traffic Management (ATM) as developed under SESAR [1] and NextGen [2] in Europe and the US, respectively. For communications, a common understanding within ICAO has been reached that a single data link technology is not capable of covering the communication needs for all phases of flight. Therefore, the Future Communications Infrastructure (FCI) [3] has been developed comprising a set of data link technologies for aeronautical communications. For the airport,
AeroMACS (Aeronautical Mobile Airport Communications System) is currently developed within NextGen and SESAR which is strongly based on the WiMAX standard. The European Space Agency (ESA) initiated the development of a future satellite-based communications system for aviation within their ESA Iris program [4], supplemented by work performed within SESAR. For air/ground communications, currently two candidate systems are under consideration for the L-band Digital Aeronautical Communication System (LDACS) [5]. LDACS1 employs a broadband transmission using Orthogonal Frequency-Division Multiplexing (OFDM), whereas LDACS2 is a narrowband single-carrier system. Current work within SESAR is devoted to develop a proposal for the selection of the LDACS technology to be standardized by ICAO.

For navigation, ICAO recommends the further development of GNSS (Global Navigation Satellite System) based technologies as primary means for navigation. It is envisaged to use GNSS not only for area navigation but also for approach, take-off, and landing. To achieve the required navigation performance in terms of precision, continuity, and integrity augmentation systems are used to assist GNSS, e.g. SBAS (Satellite Based Augmentation System), A-RAIM (Advanced Receiver Autonomous Integrity Monitoring) or GBAS (Ground Based Augmentation System). This way, GNSS based navigation is expected to cover even CAT III landings in the future.

The drawback of GNSS is its inherent single point of failure - the satellite. In addition, the received power of GNSS signals on ground is very low and, thus, might be easily jammed either intentional or unintentional by terrestrial systems. An example, how easily GNSS signals might be jammed is the recently installed GBAS station at Newark Liberty International Airport. GPS jammers used by truck drivers on the nearby highway caused the GBAS station to continuously shut itself down ceasing its intended operation. The GPS jammers 
are used by the truck drivers to disable the tracking devices installed by their companies. GPS jammers are inexpensive and can be purchased easily from the internet.

Navigation services must be available with sufficient performance for all phases of flight. Therefore, an alternative solution for the GNSS failure case has to be available, known as APNT (Alternative Positioning, Navigation, and Timing). A possible APNT solution is to increase the density of DME (Distance Measuring Equipment) stations and perform multilateration with the DME signals for navigation. However, this approach has two main disadvantages. Firstly, it requires a costly extension of the DME infrastructure. Secondly, this approach might have a severe impact on the sustainable use of the L-band for communications as foreseen within ICAO. The L-band will be used more intensively by DME than today making it much more complicated to find sufficient spectrum resources to cover the growing communications demand expected on a mid- and long-term perspective.

Another approach towards APNT is to integrate a navigation function into the new L-band communication system, e.g. into LDACS1. This way, APNT is covered by LDACS1 and additional DME ground stations are not necessary. The ground infrastructure for APNT is deployed through the implementation of LDACS1 ground stations. In addition, if it is shown that LDACS1 can reliably cover the navigation function, an extension of the DME infrastructure for APNT is not necessary and even partial removal of DME ground stations might be possible. In this case, the L-band spectrum available for communications is increased and a sustainable use of the L-band for communications is assured.

This paper reports on the proposal to use LDACS1 for navigation and presents first results about the feasibility of this approach. The reminder of this paper is organized as follows. In Section 2, LDACS1 is briefly reviewed and Section 3 describes how ranging with LDACS1 is performed. The construction of position information from the obtained ranging measurements is given in Section 4. A first performance assessment of positioning with LDACS1 is presented in Section 5 for two different scenarios. Finally, in Section 6 some conclusion are drawn and an outlook on future work is given.

\section{LDACS1 System Overview}

In this section, we will give a short overview of the LDACS1 system, focusing on the system characteristics being relevant for navigation based on the LDCAS1 signal. For more detailed information, please refer to the LDACS1 system specification [6].

\section{Main System Capabilities}

LDACS1 is intended to operate in the lower part of the L-band (960-1164 MHz). It is designed as a Frequency-Division Duplex (FDD) system, which enables a Ground Station (GS) to transmit continuously at a certain frequency, while the Airborne Stations (AS) transmit at the same time but at a different frequency. For LDACS1, the frequency spacing between Forward Link (FL, from GS to AS) and the Reverse Link (RL, from AS to GS) is proposed to be $63 \mathrm{MHz}$.

For LDACS1 deployment in L-band different scenarios are possible [6]. The most interesting approach is the inlay scenario where the LDACS1 channels with a bandwidth of approximately 500 $\mathrm{kHz}$ are placed in between the existing DME channel grid of $1 \mathrm{MHz}$ with an offset of $500 \mathrm{kHz}$ to the DME center frequencies. This approach allows LDACS1 deployment without changing DME assignments. For the inlay scenario the frequency range from 985.5 to $1008.5 \mathrm{MHz}$ for the $\mathrm{FL}$ is foreseen whereas the RL should be placed in the frequency range from 1048.5 to $1071.5 \mathrm{MHz}$. This choice minimizes the mutual interference between LDACS1 and other L-band systems, mainly the Secondary Surveillance Radar (SSR) Mode S and the Universal Access Transceiver (UAT).

The LDACS1 signal is a multi-carrier signal, based on OFDM technology. For navigation purposes, an AS has to exploit the signals from different GS, thus, we will focus on the FL in the following. How the AS can exploit the signal from a certain GS, and how the measurements from different GS can be combined, will be explained later on. 
In the FL, LDACS1 operates as a cellular point-to-multipoint system. As depicted in Figure 1, a star-topology is assumed, where all AS within a certain volume of space, called the LDACS1 cell, are connected to the controlling GS. The LDACS1 GS is a centralized instance that controls LDACS1 communications within the cell.

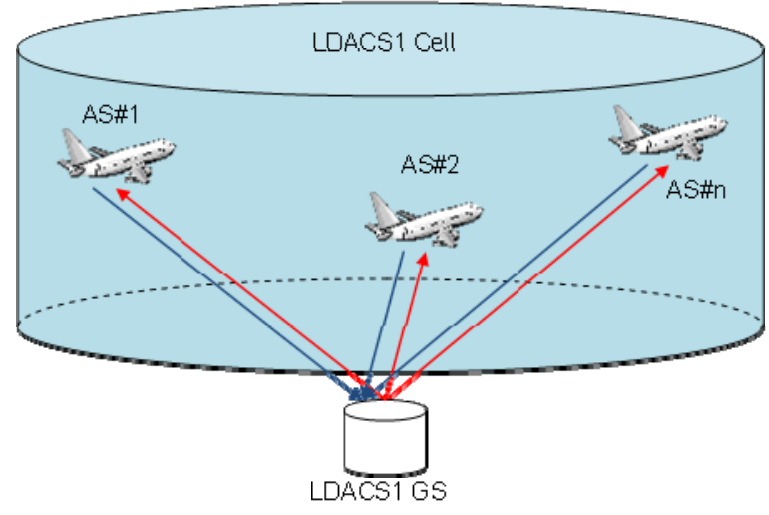

Figure 1. LDACS1 cell topology.

Adjacent cells use different LDACS1 channels with fixed center frequencies. That way, the transmission signals from different GS can be easily distinguished and separated at the AS for both communication and navigation purposes. A critical issue might be the signal quality of the different received signals at an AS from different GS. Especially if an AS is in the center of a cell, the fairly high distance to GS of neighboring cells might lead to a low Signal-to-Noise Ratio (SNR) of the received signal, due to large free-space losses. This issue is not further addressed in our paper, as we perform a first feasibility study on LDACS1 navigation rather than presenting an already mature navigation system design. If required, this issue might be resolved, e.g. by applying antenna beamforming.

The cellular approach, where neighboring LDACS1 cells use different LDACS1 channels, imposes some constraints onto the navigation capabilities of LDACS1. While it is trivial to exploit the signal from the controlling GS for ranging, it is not possible to measure signals from neighboring GS at exactly the same timing instance unless multiple receiving frontends are employed at the AS. Possibilities how measurements to different GS without the use of multiple receiving frontends might be performed are presented in the next subsection where the LDACS1 framing structure is discussed in detail.

\section{LDACS1 Framing}

The LDACS1 framing structure for FL and RL is based on Super-Frames (SF) of length $T_{\mathrm{SF}}=240 \mathrm{~ms}$ which repeat periodically. For the navigation functionality, we are interested in the continuous FL transmission, where the SF consists of a Broadcast (BC) frame followed by four MultiFrames (MF). Each MF itself contains nine Data/CC frames. The mapping of either user data or common control information is out of scope for our investigation, as this does not influence the navigation capabilities. The SF structure is depicted in Figure 2.

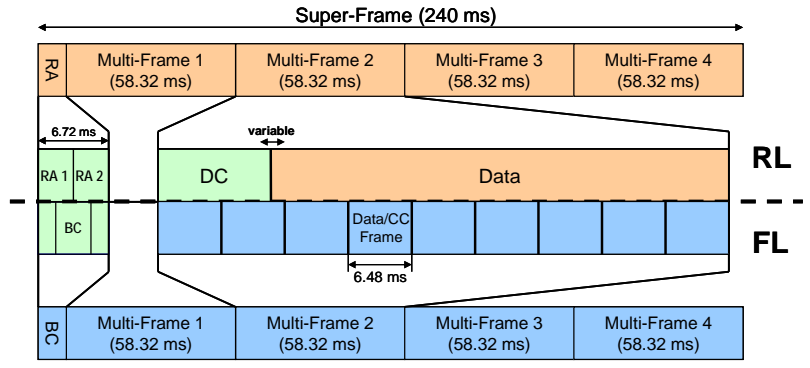

Figure 2. LDACS1 framing structure.

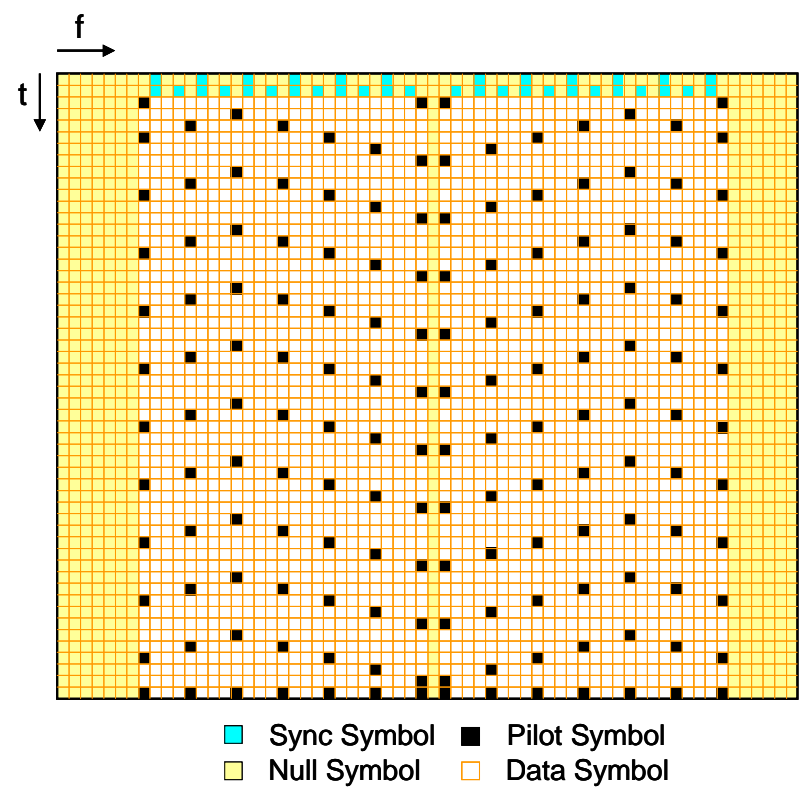

Figure 3. Structure of Data/CC frame. 
When using LDACS1 for navigation purposes, one has to exploit the two synchronization symbols which are inserted at the beginning of each Data/CC frame. The Data/CC frame structure including synchronization, null, pilot, and data symbols is depicted in Figure 3.

The duration of a FL Data/CC frame is $T_{\text {Data/CC }}=6.48 \mathrm{~ms}$, leading to a navigation update opportunity each $6.48 \mathrm{~ms}$. Additional synchronization symbols are placed in the BC frame, which is subdivided into three sub-frames $\mathrm{BC} 1, \mathrm{BC} 2$ and $\mathrm{BC} 3$, as depicted in Figure 4. The duration of $\mathrm{BCl}$ and $\mathrm{BC} 3$ is $T_{\mathrm{BC} 1 / 3}=1.8 \mathrm{~ms}$ whereas for $\mathrm{BC} 2$ it is $T_{\mathrm{BC} 2}=3.12 \mathrm{~ms}$.

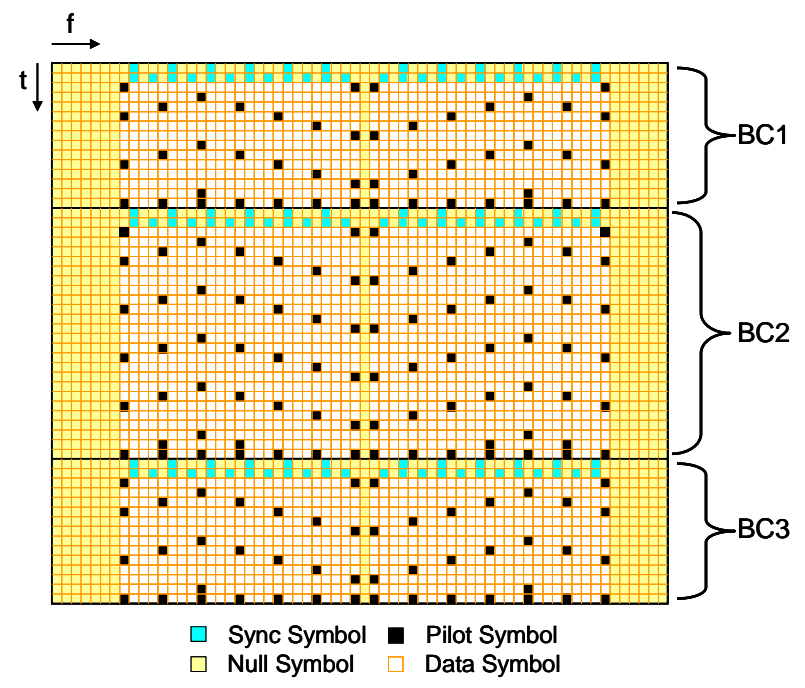

Figure 4. Broadcast frame structure.

Each of the BC sub-frames starts with two synchronization symbols which have the same structure as in the Data/CC frames. While the synchronization symbols in the Data/CC frames can only be used to obtain information about the controlling GS, the BC frame can be used to sound into neighboring cells, i.e. obtain information from different GS. However, with the existing framing structure, one could only obtain information from non-controlling GS every $240 \mathrm{~ms}$. If information from two adjacent GS is required, an update would be available each $480 \mathrm{~ms}$. This is a critical issue, as an AS, traveling with $\mathrm{MACH}$ 1, would pass around $165 \mathrm{~m}$ within one update period. To overcome this problem, one could consider modifying the framing structure, e.g. providing the possibility to obtain measurements from more than one neighboring GS in one $\mathrm{BC}$ frame. Another way could be to sound for a complete MF into different neighboring cells.

The structure of the two synchronization symbols in the frequency domain is depicted in Figure 5.

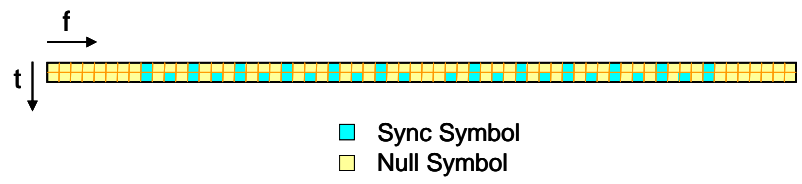

Figure 5. Synchronization symbols in frequency domain.

Due to the properties of the Fourier transform, the first synchronization symbol consists of four identical parts in the time domain and the second synchronization symbol consists of two identical parts in the time domain, as depicted in Figure 6.

\begin{tabular}{|c|c|c|c|c|c|c|c|}
\hline CP & B & B & B & B & CP & A & A \\
\hline
\end{tabular}

Figure 6. Synchronization symbols in time domain.

This structure can be exploited in the receiver to derive estimates about the receiving time of the signals from different GS, which will be explained in the next section.

\section{Ranging with LDACS1}

Our approach for positioning with LDACS1 is based on ranging from an AS to different GS. These distance estimates can be derived from the propagation duration of the LDACS1 signal from GS to AS and the propagation velocity of radio signals in the air, which is $c=3 \cdot 10^{8} \mathrm{~m} / \mathrm{s}$. For determining the propagation duration it is important to have knowledge as precise as possible about the receiving time instance and the transmission time instance. The receiving time instance can be determined easily at the AS exploiting the synchronization symbols as explained later on. For the controlling GS, the transmission time instance is inherently determined during the net entry procedure of an AS into the respective LDACS1 cell. Since all AS have to make sure that their RL transmission signals arrive synchronously at the 
GS, the GS measures the offset between GS and AS timing during the net entry procedure and reports back the obtained value to the AS [6]. The time offset value is further on continuously tracked at the AS. Since synchronized GS are assumed, the transmission time instance is valid for all GS and, thus, it is not necessary to estimate this value for GS other than the controlling GS. An alternative method to determine the transmission time instance for synchronized GS is to consider this time instance an additional variable which has to be estimated in addition to the three-dimensional AS position. Thus, a minimum of four instead of three ranging measurements is required to determine the four variables, the three coordinates of the AS position and the GS transmission time instance.

The quality of the ranging highly relies on the accuracy of the estimation of the arrival time of the signals, which complies with calculating the starting time of the $\mathrm{BC}$ and Data/CC frames by means of exploiting the synchronization symbols at the beginning of each frame. Since they are known to the aircraft in advance, they can be exploited by correlating the known time domain synchronization signal waveform with the received signal, or by correlating parts of the received signal with each other, due to the repetitive structure, as proposed in [7]. Both approaches lead to a metric, where a peak indicates the starting time of the frame. A measure for the accuracy of this approach is the variance of the timing estimate $\hat{\tau}$. The maximum achievable performance for this variance can be given by the Cramer-Rao Lower Bound (CRLB). For an OFDM system, it is defined by [8]

$$
\operatorname{var}_{\mathrm{CRLB}}(\hat{\tau})=\frac{N_{\mathrm{FFT}} N_{\text {used }}}{8 \mathrm{SNR} \pi^{2} \sum_{k=-N_{\text {used }} / 2+1}^{N_{\text {used }} / 2} k^{2}|H(k)|^{2}}
$$

with $N_{\text {FFT }}$ the size of the Fourier transformation, $N_{\text {used }}$ the number of used subcarriers in the synchronization symbol, SNR the signal-to-noise ratio of the received signal, and $H(k)$ the channel coefficient effective to subcarrier $k$.

This variance can be further reduced by averaging over several synchronization symbols. However, the AS position changes from synchronization symbol to synchronization symbol, leading to an average timing estimation. Consequently, the choice of the number of synchronization symbols used to obtain one estimate has to be determined in dependence of the speed of an AS and the target accuracy, to get a good trade-off between estimation precision and position changes within the averaging period. Table 1 summarizes maximum possible position changes for typical synchronization symbol distances in the LDACS1 frame structure, assuming an AS velocity of MACH 1.

Table 1. Position Changes for Selected Periods

\begin{tabular}{|l|r|r|}
\hline $\begin{array}{l}\text { Synchronization } \\
\text { Symbol distance }\end{array}$ & $\begin{array}{l}\text { Time } \\
\text { Period }\end{array}$ & $\begin{array}{l}\text { Position } \\
\text { change }\end{array}$ \\
\hline $\begin{array}{l}\text { First Sync Symbol } \rightarrow \\
\text { Second Sync Symbol }\end{array}$ & $120 \mu \mathrm{s}$ & $0.041 \mathrm{~m}$ \\
\hline $\begin{array}{l}\text { Data/CC Frame } \rightarrow \\
\text { Data/CC Frame }\end{array}$ & $6.48 \mathrm{~ms}$ & $2.2 \mathrm{~m}$ \\
\hline $\mathrm{MF} \rightarrow \mathrm{MF}$ & $58.32 \mathrm{~ms}$ & $20 \mathrm{~m}$ \\
\hline $\mathrm{SF} \rightarrow \mathrm{SF}$ & $240 \mathrm{~ms}$ & $82.3 \mathrm{~m}$ \\
\hline $\mathrm{BC} 1 \rightarrow \mathrm{BC} 2 \rightarrow \mathrm{BC} 3$ & $4.92 \mathrm{~ms}$ & $1.69 \mathrm{~m}$ \\
\hline
\end{tabular}

Besides the CRLB, it is of interest how close this bound can be reached by applying a realistic metric. For an Additive White Gaussian Noise (AWGN) channel, a normalized correlation between the received signal and the known transmit sequence will lead to the best result, as it exploits the full knowledge about the synchronization sequences. However, under poor channel conditions, e.g. caused by multipath propagation, the correlation between transmitted and received sequences will degrade rapidly. In this case, a correlation between received parts, corresponding to the length and the distance between the identical transmitted parts of the synchronization sequence [7], will lead to better results, as they are impaired in the same way. However, this method does not exploit the knowledge about the transmitted synchronization sequences. For an aeronautical channel, with a rather strong Line-Of-Sight (LOS) path but a possibly considerable Doppler shift, a hybrid approach might lead to good results. In a first step, the Doppler shift is estimated by the correlation between parts of the received signal. After having compensated this Doppler shift, an additional correlation between the compensated 
received signal and the known time-domain synchronization symbols will help to refine the synchronization result.

\section{Positioning Based on Ranging}

Once the ranges to multiple base stations have been computed, it is possible to estimate the position of the aircraft by means of multilateration. Assuming that the three-dimensional coordinates of the aircraft are to be estimated, ranges to at least three GS are required. In general, including more GS will further increase the positioning accuracy.

Which GS are used for positioning has a large impact on the accuracy that can be achieved. Although the estimate can always be improved by considering a larger number of GS, this is not possible from a practical point of view.

At the moment, we select GS based only on their SNR, since a higher SNR implies a better ranging estimate. However, the relative positions of the GS are just as important for the accuracy of the positioning estimate. This effect is referred to as Geometric Dilution of Precision (GDOP). More advanced techniques for the selection of GS are an important topic for future work.

We consider two different methods for the positioning. The first method is snapshot based and takes into account only the current ranging measurements. In this case, the navigation equations can be solved by the iterative GaussNewton method. However, the snapshot based approach does not consider the strong correlation between subsequent positioning estimates. Therefore, we also consider a second approach using an Extended Kalman Filter. We expect that this will improve the positioning accuracy by exploiting the correlation between subsequent positioning estimates.

All calculations are performed in an aircraftcentric Cartesian coordinate system. The z-axis is orthogonal to the Earth's surface, and the x- and yaxes span a plane tangent to the Earth's surface directly below the aircraft. Let the position of the $i$ th GS be given by $\mathbf{p}_{G S, i}=\left(x_{G S, i}, y_{G S, i}, z_{G S, i}\right)^{T}$. Note that all vectors are denoted by bold lower case letters, and all matrices by bold upper case letters. The ranging measurements to the GS are collected in the vector $\hat{\mathbf{d}}$. These are related to the true distances according to the measurement model

$$
\hat{\mathbf{d}}=\mathbf{d}\left(\mathbf{p}_{A S}\right)+\mathbf{n},
$$

where $\mathbf{p}_{A S}$ is the true position of the aircraft. The true distances from $\mathbf{p}_{A S}$ to the GS are collected in the vector

$$
\mathbf{d}\left(\mathbf{p}_{A S}\right)=\left(\left\|\mathbf{p}_{A S}-\mathbf{p}_{G S, 1}\right\|, \ldots,\left\|\mathbf{p}_{A S}-\mathbf{p}_{G S, N}\right\|\right)^{T}
$$

and $\boldsymbol{n}$ is the vector of the measurement error of the range to each of the base stations, which is assumed zero-mean AWGN with diagonal covariance matrix

$$
\boldsymbol{\Sigma}=\mathrm{E}\left\{\mathbf{n n}^{T}\right\},
$$

where $E\{$.$\} denotes expectation. Thus, the$ diagonal elements of $\boldsymbol{\Sigma}$ are the variances of the ranging estimates from the AS to the different GS. A lower bound on these variances is given by the CRLB according to in Eq. (1). If real variances should be considered, the Minimum Squared Error (MSE) of the ranging estimates obtained from the applied synchronization procedure have to be taken.

\section{Weighted Least Squares Position Estimation}

The aircraft position can be efficiently estimated in the Weighted Least Squares (WLS) sense in an iterative manner by means of the GaussNewton method, where the contribution of each GS is weighted according to the inverse of the variance of its ranging estimate [8]. This will assign more weight to those ranging estimates with lower variance, i.e. higher reliability. The WLS estimate of the aircraft position $\hat{\mathbf{p}}_{A S}$ is given by

$$
\hat{\mathbf{p}}_{A S}=\arg \min _{\mathbf{p}}(\mathbf{d}(\mathbf{p})-\hat{\mathbf{d}})^{T} \boldsymbol{\Sigma}^{-1}(\mathbf{d}(\mathbf{p})-\hat{\mathbf{d}}),
$$

where $\boldsymbol{\Sigma}$ is the diagonal covariance matrix from Eq. (4), $\hat{\mathbf{d}}$ is the vector of ranging estimates to the GS, and $\mathbf{d}(\mathbf{p})$ is the vector of the calculated distances $d_{i}(\mathbf{p})$ from an arbitrary point $\mathbf{p}=(x, y, z)^{T}$ to GS $i$. This results in the update rule for the position estimate of the aircraft for iteration step $k+1$ 


$$
\begin{gathered}
\hat{\mathbf{p}}_{A S, k+1}=\hat{\mathbf{p}}_{A S, k}+\left(\boldsymbol{\Phi}^{T}\left(\hat{\mathbf{p}}_{A S, k}\right) \boldsymbol{\Sigma}^{-1} \boldsymbol{\Phi}\left(\hat{\mathbf{p}}_{A S, k}\right)\right)^{-1} \bullet \\
\boldsymbol{\Phi}^{T}\left(\hat{\mathbf{p}}_{A S, k}\right) \boldsymbol{\Sigma}^{-1}\left(\mathbf{d}\left(\hat{\mathbf{p}}_{A S, k}\right)-\hat{\mathbf{d}}\right),
\end{gathered}
$$

where $\boldsymbol{\Phi}(\mathbf{p})$ is the Jacobian matrix defined as

$$
\begin{aligned}
& \boldsymbol{\Phi}(\mathbf{p})=\left[\frac{\delta}{\delta x}, \frac{\delta}{\delta y}, \frac{\delta}{\delta z}\right]^{T} \otimes \mathbf{d}(\mathbf{p}) \\
& =\left(\begin{array}{ccc}
\frac{x-x_{G S, 1}}{d_{1}(\mathbf{p})} & \frac{y-y_{G S, 1}}{d_{1}(\mathbf{p})} & \frac{z-z_{G S, 1}}{d_{1}(\mathbf{p})} \\
\vdots & \vdots & \vdots \\
\frac{x-x_{G S, N}}{d_{N}(\mathbf{p})} & \frac{y-y_{G S, N}}{d_{N}(\mathbf{p})} & \frac{z-z_{G S, N}}{d_{N}(\mathbf{p})}
\end{array}\right)
\end{aligned}
$$

and " $\otimes$ " denotes the Kronecker product. Due to the structure of the update rule, only a matrix of size $3 \times 3$ needs to be inverted in each update step, regardless of the number of GS being considered. The Gauss-Newton method converges very fast and provides accurate position estimates for good GDOP and initial values. Choosing the initial position as the center of mass of all GS positions has shown to provide good results in general. However, poorly chosen initial values or situations with high GDOP may prevent the Gauss-Newton method from converging properly. The convergence properties can be improved by introducing a regularization term, as proposed by Levenberg [9] and Marquardt [10].

\section{Extended Kalman Filter}

The WLS approach described above considers each set of ranging measurements independently, and does not take into account the correlation between subsequent measurements. This additional information can be exploited by a Kalman filter. A classical linear Kalman Filter would take the position estimates provided by the snapshot method as input and perform a kind of smoothing, based on the aircraft mobility model. In contrast, an Extended Kalman Filter (EKF) [8] is a nonlinear filter that can operate directly on the ranging estimates to the GS. The basic operation of a Kalman filter is as follows. In a prediction phase, the filter predicts the system state in time step $k$ using the state estimate from the previous time step $k-1$. In the following update phase, the prediction is adjusted, based on the current measurement.

In our case, the state vector $\mathbf{s}_{k}$ for time step $k$, that is to be estimated, comprises the aircraft position $\mathbf{p}_{A S, k}=\left(x_{A S, k}, y_{A S, k}, Z_{A S, k}\right)^{T}$ as well as its velocity $\mathbf{v}_{k}=\left(v_{x, k}, v_{y, k}, v_{z, k}\right)^{T}$ and, thus, results in $\mathbf{s}_{k}=\left[x_{A S, k}, y_{A S, k}, z_{A S, k}, v_{x, k}, v_{y, k}, v_{z, k}\right]^{T}$.

This vector is assumed to change over time according to the underlying linear state model

$\mathbf{s}_{k}=\mathbf{A}_{k-1}\left(\mathbf{s}_{k-1}\right)+\boldsymbol{\eta}_{k-1}$,

where in general the state matrix $\mathbf{A}_{k-1}$ is time variant. However, for our purposes a very simple, constant mobility model for the aircraft with time invariant state matrix $\mathbf{A}$ is sufficient, given by

$$
\mathbf{A}=\left[\begin{array}{llllll}
1 & 0 & 0 & T & 0 & 0 \\
0 & 1 & 0 & 0 & T & 0 \\
0 & 0 & 1 & 0 & 0 & T \\
0 & 0 & 0 & 1 & 0 & 0 \\
0 & 0 & 0 & 0 & 1 & 0 \\
0 & 0 & 0 & 0 & 0 & 1
\end{array}\right],
$$

where $T$ is the update rate of the Kalman filter. The state noise $\boldsymbol{\eta}_{k-1}$ specifies the unknown change in the aircraft position and velocity in $x-, y-$, and $z-$ direction. This uncertainty can be modeled based on the flight performance of the aircraft, i.e. maximum climb, turn, or acceleration rates. As in the previous section, the measurements are the measured ranges to the GS, contained in the vector $\hat{\mathbf{d}}_{k}$. The time dependent, nonlinear measurement model is then given by

$\hat{\mathbf{d}}_{k}=\mathbf{d}\left(\mathbf{p}_{A S, k}\right)+\mathbf{n}_{k}$.

The vector $\mathbf{p}_{A S, k}$ is the true position of the aircraft in time step $k$, and corresponds to the first three components of the state vector. The last three components of the state vector, i.e. the aircraft velocities, are treated as hidden state variables and 
do not contribute to the observation $\hat{\mathbf{d}}_{k}$. The state estimate of the EKF in step $k$ is given by

$\hat{\mathbf{s}}_{k \mid k}=\hat{\mathbf{s}}_{k \mid k-1}+\mathbf{K}_{k}\left(\hat{\mathbf{d}}_{k}-\mathbf{d}\left(\hat{\mathbf{p}}_{A S, k \mid k-1}\right)\right)$,

where $\hat{\mathbf{s}}_{k \mid k-1}=\mathbf{A} \hat{\mathbf{s}}_{k-1 \mid k-1}$ is the prediction of the current state based on the previous state estimate, $\hat{\mathbf{p}}_{A S, k \mid k-1}$ contains the first three components of $\hat{\mathbf{s}}_{k \mid k-1}$, i.e. the predicted AS position, and $\mathbf{K}_{k}$ is the Kalman gain matrix, which is calculated as

$$
\begin{aligned}
\mathbf{K}_{k} & =\mathbf{M}_{k \mid k-1} \mathbf{H}_{k}^{T}\left(\hat{\mathbf{p}}_{A S, k \mid k-1}\right) \cdot \\
& \left(\boldsymbol{\Sigma}_{k}+\mathbf{H}_{k}\left(\hat{\mathbf{p}}_{A S, k \mid k-1}\right) \mathbf{M}_{k \mid k-1} \mathbf{H}_{k}^{T}\left(\hat{\mathbf{p}}_{A S, k \mid k-1}\right)\right)^{-1},
\end{aligned}
$$

where $\mathbf{M}_{k \mid k-1}$ is the covariance matrix of the prediction of the state vector, $\boldsymbol{\Sigma}_{k}$ is the covariance matrix of the measurement noise $\mathbf{n}_{k}$, and $\mathbf{H}_{k}\left(\hat{\mathbf{p}}_{A S, k \mid k-1}\right)$ is the Jacobian matrix

$\mathbf{H}_{k}\left(\mathbf{p}_{k}\right)=\frac{\partial \mathbf{d}_{k}\left(\mathbf{p}_{k}\right)}{\partial \mathbf{s}_{k}}$,

evaluated at $\mathbf{p}_{k}=\hat{\mathbf{p}}_{A S, k \mid k-1}$ which is quite similar to the Jacobian matrix $\boldsymbol{\Phi}(\mathbf{p})$ in the Gauss-Newton method of the previous section. The exact expressions for these matrices are omitted herein, but their calculation is straightforward, and the interested reader is referred to [8].

As stated above, the EKF requires a mobility model for the aircraft, specifying the maximum acceleration in $\mathrm{x}-\mathrm{y}$ - and $\mathrm{z}$-direction. By choosing lower values, the EKF will allow smaller changes in the position estimate of the aircraft from step to step, and the estimate will be smoothed significantly. For very high values, the EKF will tend towards the snapshot based position estimate. We have derived numerical values for the acceleration in each of the three directions by analyzing an aircraft trajectory file of typical departures and arrivals at Frankfurt airport. To account for unexpected behavior, an additional margin was added to the maximum values encountered in the file.
The EKF requires one matrix inversion in every update of the Kalman gain matrix. In contrast to the Gauss-Newton method of the previous section, the dimensions of this matrix increase with the number of GS being considered.

\section{Performance Assessment}

\section{Simulation Scenarios}

The positioning accuracy of the proposed system depends on the actual number and distribution of LDACS1 GS. Since such information is currently not available, exact numbers for the expected performance are difficult to provide. For our performance analysis, we have assumed the current locations of DME stations in Germany as locations of LDACS1 GS. Although this will not necessarily reflect the true deployment of LDACS1 base stations in the future, it does allow for a more fair comparison between the positioning performance of the proposed system and what might be achieved by DME.

In order to assess the possible performance that can be achieved by using the LDACS1 system for positioning, we have simulated two different flight scenarios.

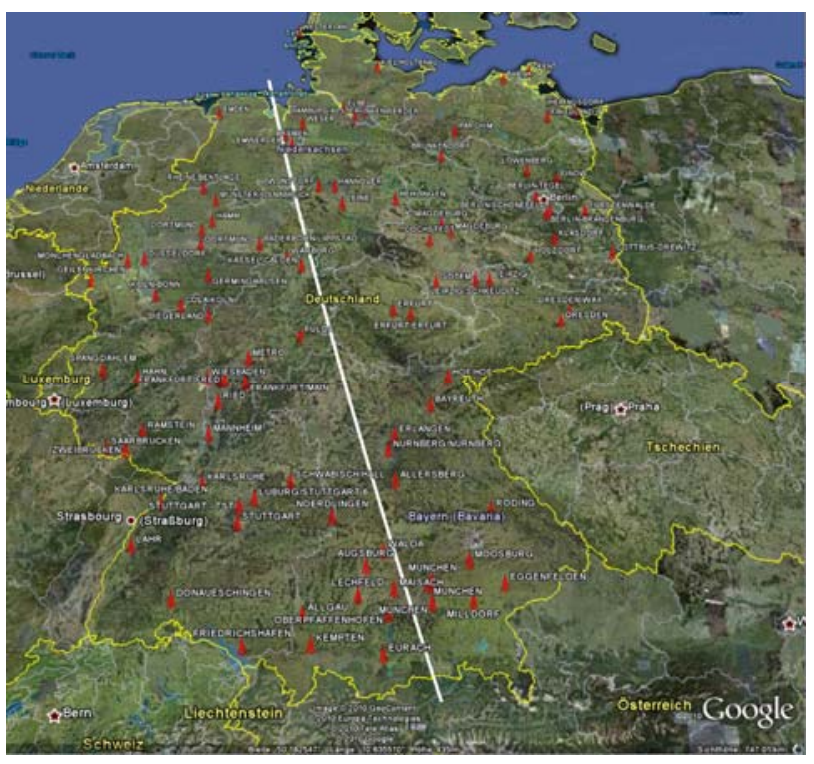

Figure 7. Flight route for scenario 1, as well as GS positions.

The first scenario considers an aircraft flying across Germany from the North Sea to the Austrian 
border along a great circle route at a constant altitude of $10000 \mathrm{~m}$. Positioning performance will fluctuate due to the irregular distribution of GS. Since we are only considering GS within Germany, fewer stations will be visible to the aircraft at the beginning and the end of the simulated flight path. The route flown and the number of visible GS are shown in Figure 7 and Figure 8, respectively. The positions of the GS are also depicted in Figure 7. Note that a GS is considered to be visible if a lineof-sight path between the aircraft and the GS exists.

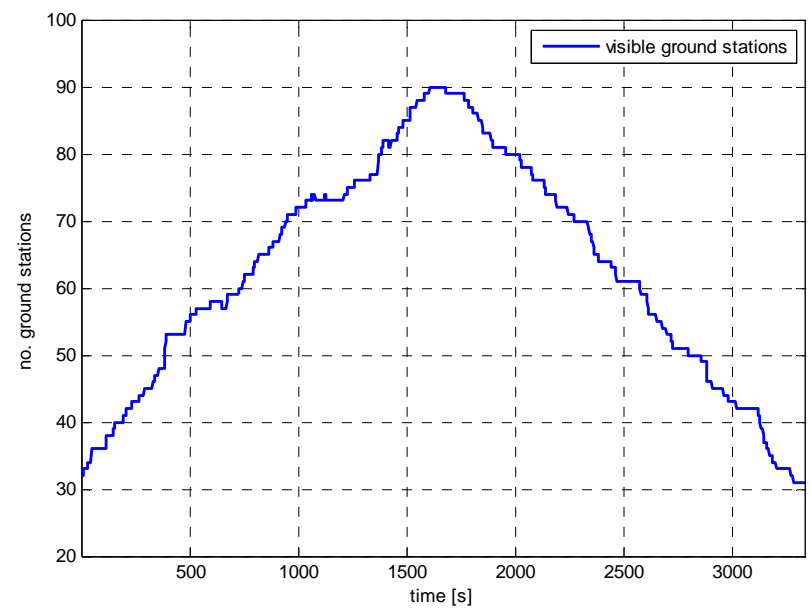

Figure 8. Number of GS visible to the aircraft in scenario 1.

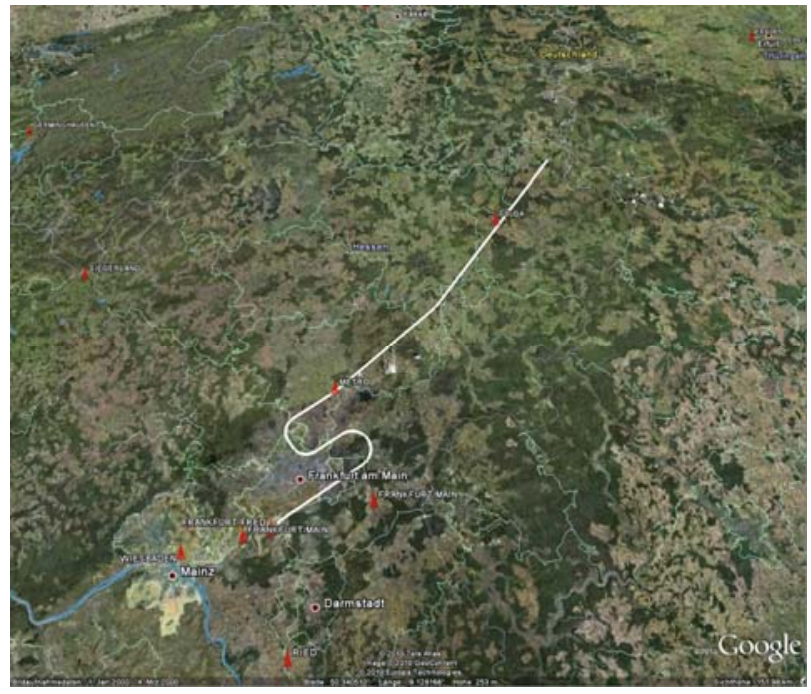

Figure 9. Flight route for scenario 2, approach into Frankfurt Airport.

The second scenario considers an aircraft arriving at Frankfurt International Airport, using an actual arrival route and realistic aircraft flight performance data. As the aircraft descends, the number of visible GS will steadily decrease. The flight route, the number of visible GS, and the altitude profile flown during the approach are shown in Figure 9, Figure 10, and Figure 11, respectively. It can be seen, that the number of visible GS decreases considerably (from around 25 to 6) as the aircraft descends towards the airport.

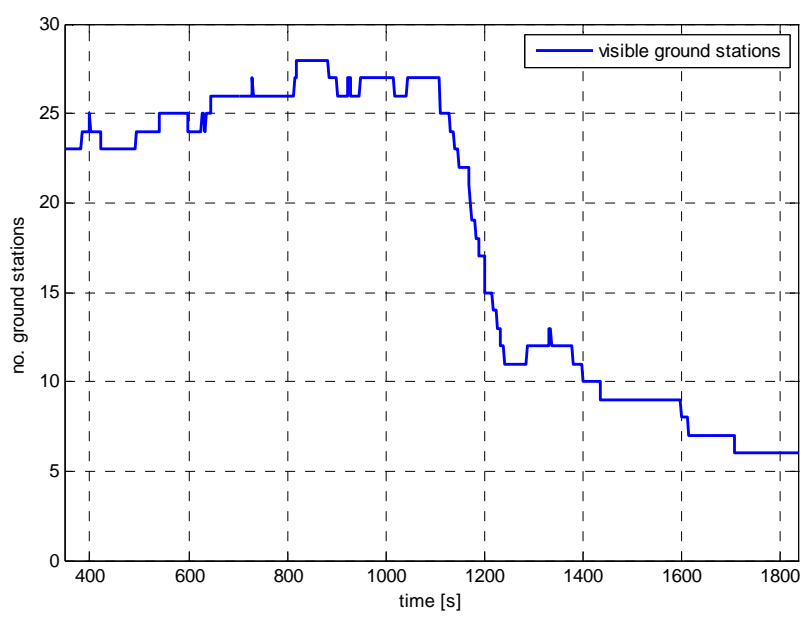

Figure 10. Number of GS visible to the aircraft in scenario 2.

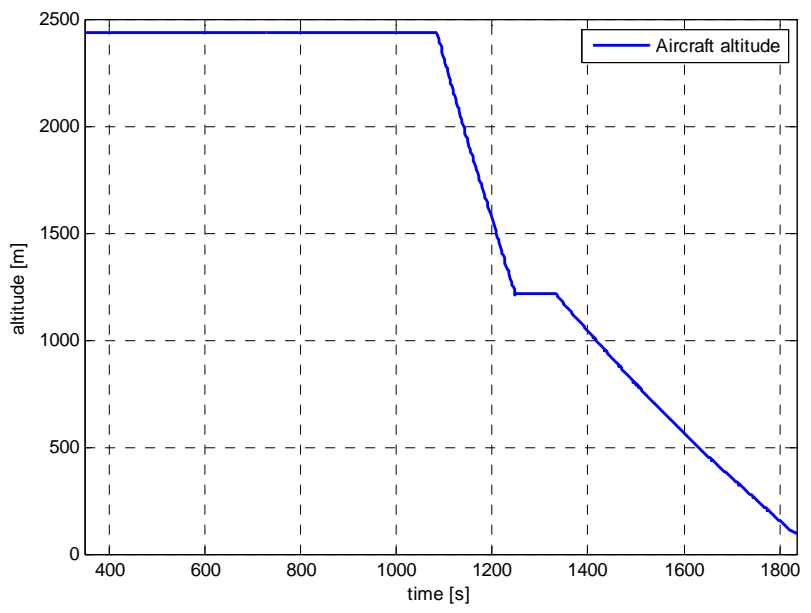

Figure 11. Altitude profile of the aircraft in scenario 2.

\section{Simulation Results}

For both flight scenarios, simulations are performed where ranging estimates are collected every two seconds. For simplicity, the selection of GS used for ranging is solely based on the SNR, i.e. the GS producing the highest SNR at the AS are 
selected. Other criteria, like the geometric distribution of GS, are not taken into account. Either three or ten GS from the set of visible GS are considered for obtaining position estimates from the measured ranges. Three is the minimal required number of GS for three-dimensional position estimation. Ten GS are chosen to show the improvement possible when more than three GS are considered or when GS with very low GDOP are used for ranging, since it can be assumed that within 10 GS there is an ensemble of three GS with low GDOP.

For all simulations the positioning error serves as performance measure. Four different presentations of the position error are applied to visualize the simulation results. The first two present the position error as deviation of the estimated position from the real position according to the two methods described in Section 3, i.e. presentation one uses the snapshot based WLS minimization via the Gauss-Newton method and presentation two the EKF method. The other two presentations provide lower bounds on the position error based on the CRLB for the position estimate $\hat{\mathbf{p}}_{\text {AS }}$ according to

$$
\operatorname{CRLB}\left(\hat{\mathbf{p}}_{A S}\right)=\sqrt{\operatorname{tr}\left(\boldsymbol{\Phi}^{\mathrm{T}}\left(\hat{\mathbf{p}}_{A S}\right) \boldsymbol{\Sigma}^{-1} \boldsymbol{\Phi}\left(\hat{\mathbf{p}}_{A S}\right)\right)^{-1}},
$$

where $\operatorname{tr}($.$) calculates the trace of a matrix.$ According to Eq. (4) and the explanation given thereafter in Section 4, the diagonal elements of $\boldsymbol{\Sigma}$ are the variances of the ranging estimates from the AS to the different GS. For the third presentation, the MSE of the ranging estimates obtained from the real synchronization procedure as currently applied in LDACS 1 are used as variances for the covariance matrix $\boldsymbol{\Sigma}$ and for the fourth presentation, the variances are lower bounded by the CRLB according to Eq. (1).

Figure 12 shows the positioning performance for scenario 1, using three GS and the presentations one (blue circles), two (black dots), and three (green line). As an important result, the position error stays typically below $100 \mathrm{~m}$, although only three GS are considered and GS choice is solely based on SNR. At the beginning and end of the flight segment considered here, the error is highest, since our simulation only contains GS located within Germany. Discontinuities in the CRLB (green line) are caused by changes in the set of GS being considered for ranging during the flight. In addition, it can be seen that the Gauss-Newton method does not always converge properly, e.g. between $\mathrm{t}=$ $1058 \mathrm{~s}$ and $\mathrm{t}=1328 \mathrm{~s}$. In this situation, the three GS that are selected due to their high SNR are approximately aligned in a row, leading to a high GDOP. Under normal conditions, the EKF does not appear to provide a substantial higher positioning accuracy than the Gauss-Newton method. However, the state model of the EKF does not allow arbitrary changes in the position estimate from one time step to the next. Therefore, the EKF is still able to provide a reasonable position estimate in those situations where the Gauss-Newton method has convergence problems.

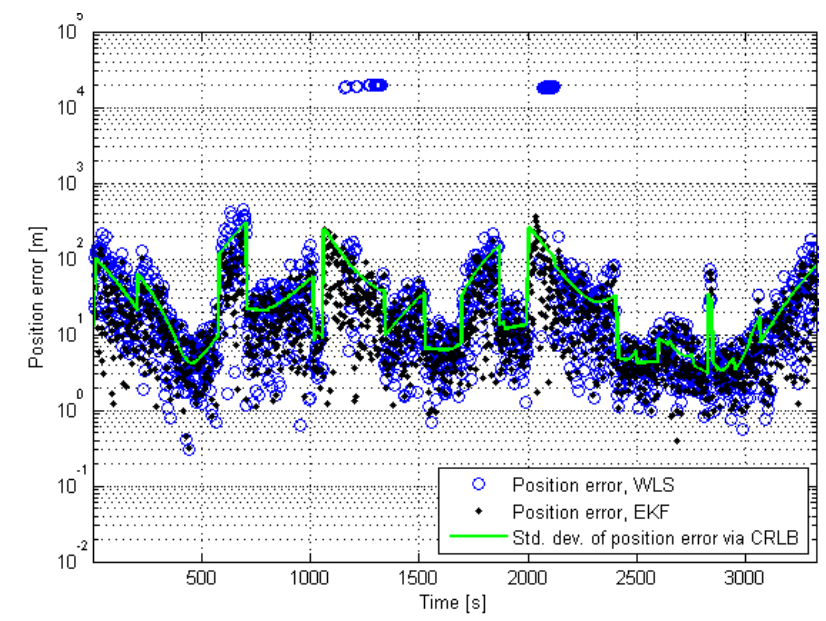

Figure 12. Positioning results for scenario 1, using three GS.

In Figure 13, again results for scenario 1 are presented but considering ten instead of three GS for ranging. In contrast to Figure 12, only the CRLB for the positioning estimate is given, i.e. presentations three (solid lines) and four (dashed lines) are applied. In addition to the CRLB of the three-dimensional position (green) the CRLB for the vertical (red) and horizontal (blue) components are depicted as well. Figure 13 reveals three interesting results. First, considering ten instead of three GS improves the positioning estimate for presentation three by roughly one order of magnitude compared to Figure 12, resulting in a position error below $10 \mathrm{~m}$. Second, the position error for the horizontal component is considerably smaller and stays in the range 1-2 m, i.e. the position error is dominated by the vertical 
component. This is due to the fact that the GS are located at a comparable altitude, leading to a significant GDOP in the vertical component. The horizontal component can typically be estimated much more accurately. Third, comparing presentations three and four, i.e. a realistic with the ideal synchronization procedure, shows that applying more sophisticated synchronization algorithms could further improve the position estimate, thereby allowing the positioning estimate to approach the dashed lines. However, the currently applied LDACS1 synchronization procedure is already quite reasonable, since improvements are restricted to roughly a factor 2-3.

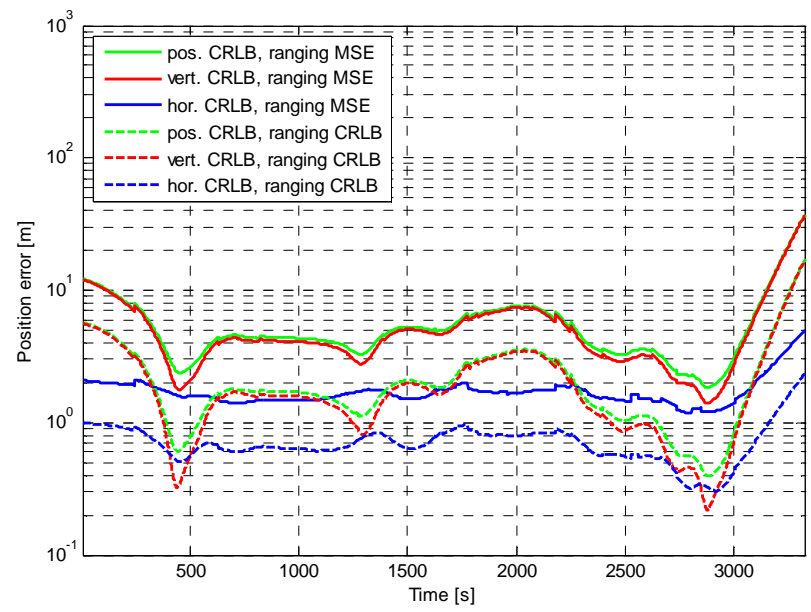

Figure 13. CRLB of positioning with ten GS for scenario 1.

Figure 14 and Figure 15 show the positioning performance for scenario 2, the approach to Frankfurt airport, for three and ten GS as in scenario 1 using exactly the same presentations as in Figure 12 and Figure 13. In this scenario, the position error typically lies below $20 \mathrm{~m}$. However, in Figure 14 the convergence problems of the Gauss-Newton method (blue circles) are particularly evident at the end of the approach. In this case, the GDOP when using only three stations is very high, and the Gauss-Newton method fails to converge during the last 200 seconds of the flight. The accuracy of the EKF (black dots) also suffers considerably, but it still manages to provide a position estimate. Using a larger number of GS or a better chosen set of GS significantly improve the position estimate as can be seen from Figure 15.
In Figure 15, the CRLB for the total position error as well as its horizontal and vertical components are presented, assuming the MSE (solid lines) and the CRLB (dashed lines) of the ranging estimate, respectively. The position error can typically be kept below $10 \mathrm{~m}$, with the error of the horizontal component as low as 1-2 m. As mentioned above, the horizontal error is typically much smaller than the vertical error. However, there are two situations (at time $\mathrm{t}=510 \mathrm{~s}$ and $\mathrm{t}=$ $960 \mathrm{~s}$ ) in which the vertical position error suddenly decreases and may even drop below the horizontal error. This effect occurs whenever the aircraft flies almost directly over a GS.

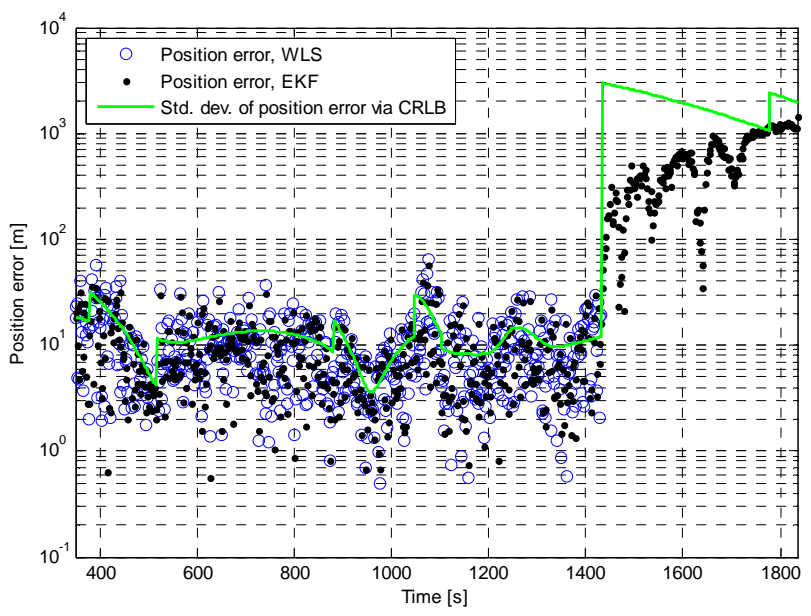

Figure 14. Positioning results for scenario 2, using three GS.

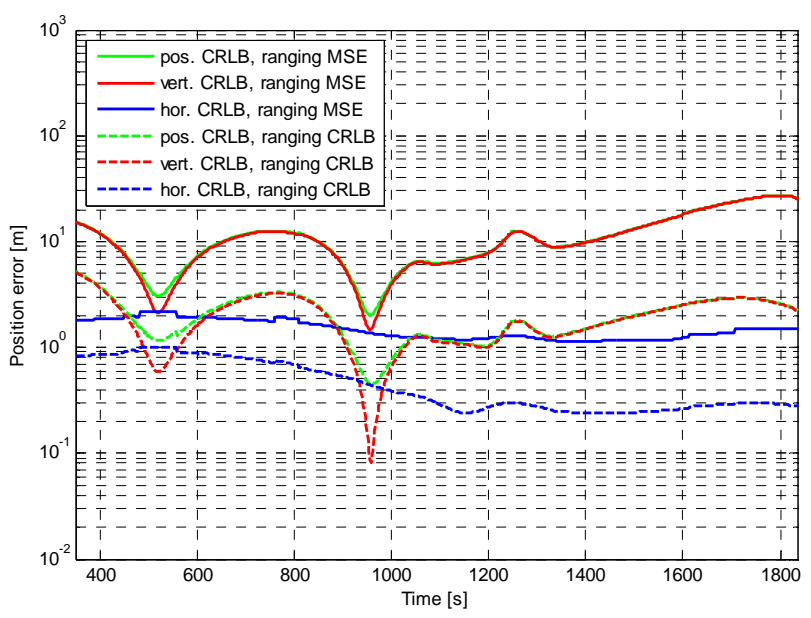

Figure 15. CRLB of positioning with ten GS for scenario 2. 


\section{Conclusion and Outlook}

In this paper, a proposal for using the future Lband communication system candidate LDACS1 for APNT is made. It is described, how LDACS1 could be used for positioning and first results about the feasibility of this approach are presented.

Even for the most challenging scenario, i.e. considering only three GS which are selected according to the SNR criteria, typically the position error already stays below $100 \mathrm{~m}$. The position accuracy is considerably increased, if more GS are used for positioning and/or their geographic locations are taken into account when selecting the GS to avoid a large GDOP. In this case, position errors below $10 \mathrm{~m}$ are achieved.

If only the horizontal components of the AS position are estimated, the position error is as low as 1-2 $\mathrm{m}$. This might be an interesting approach, since the altitude can be measured by other means, e.g. the aircraft altimeter.

Currently, additional work is performed with the goal to develop a complete navigation system design based on LDACS1. This work comprises

- the optimization of the LDACS1 synchronization procedure to better approach the lower bound for the error of the position estimate,

- the adaptation of the LDACS1 framing structure to comply with the need to measure ranges to several GS,

- the development of an algorithm for selecting the GS based not only on the SNR but also on their geometric distribution to avoid GS constellations with large GDOP.

As a final conclusion, we propose to further follow the approach of positioning with LDACS1 for APNT. The results of the performed feasibility study as presented in this paper are very promising. In addition, following this approach sustainable use of the L-band for communications as foreseen within ICAO is assured.

\section{References}

[1] http://www.sesarju.eu/

[2] http://www.faa.gov/nextgen/

[3] http://www.eurocontrol.int/communications/ public/standard_page/com_future.html

[4] http://telecom.esa.int/telecom/www/area/index. cfm? fareaid $=56$

[5] http://www.eurocontrol.int/communications/ public/standard_page/LDACS.html

[6] M. Sajatovic, B. Haindl, M. Ehammer, Th. Gräupl, M. Schnell, U. Epple, S. Brandes, February 2009, "LDACS1 System Definition Proposal: Deliverable D2", Edition 1.0, Eurocontrol Study.

[7] T. Schmidl, D. Cox, 1997, "Robust Frequency and Timing Synchronization for OFDM", IEEE Transactions on Communication, Vol. 45, no. 12.

[8] S. M. Kay, 1993, Fundamentals of Statistical Signal Processing, Vol. 1 - Estimation Theory, Prentice Hall.

[9] K. Levenberg, 1944, "A Method for the Solution of Certain Problems in Least Squares", Quarterly Journal of Applied Mathematics, vol. 2, pp. 164-168.

[10] D. Marquardt, 1963 "An Algorithm for Least Squares Estimation of Nonlinear Parameters", SIAM Journal on Applied Mathematics, vol. 11, pp. 431-441.

\section{Email Addresses}

Michael.Schnell@,DLR.de

Ulrich.Epple@DLR.de

Felix.Hoffmann@DLR.de

2011 Integrated Communications Navigation and Surveillance (ICNS) Conference

May 10-12, 2011 